\title{
Reliability of percentage ideal weight for height
}

\author{
Vanessa J Poustie, Ruth M Watling, Deborah Ashby, Rosalind L Smyth
}

\begin{abstract}
Aims-To assess the reliability of percentage ideal weight for height (\%WFH) as a measure in children.

Methods-Forty two dietitians calculated $\%$ WFH of six children. Eleven of the 42 repeated the calculations.

Results-Interexaminer estimates varied by 16.5 to 40 percentage points (mean 27.8). Intraexaminer variability was also large.

Conclusions-\%WFH is an unreliable measure of nutritional status.

(Arch Dis Child 2000;83:183-184)
\end{abstract}

Health, Royal

Liverpool Children's

Hospital, Alder Hey,

Liverpool L12 2AP, UK

V J Poustie

R L Smyth

\section{Department of \\ Dietetics and \\ Nutrition, Royal \\ Liverpool Children's \\ Hospital \\ R M Watling \\ Wolfson Institute of Preventive Medicine, Queen Mary \& \\ Westfield College, \\ London EC1M 6BQ, \\ UK \\ D Ashby}

Correspondence to:

Professor Smyth

email: r.1.smyth@liv.ac.uk

Accepted 21 March 2000

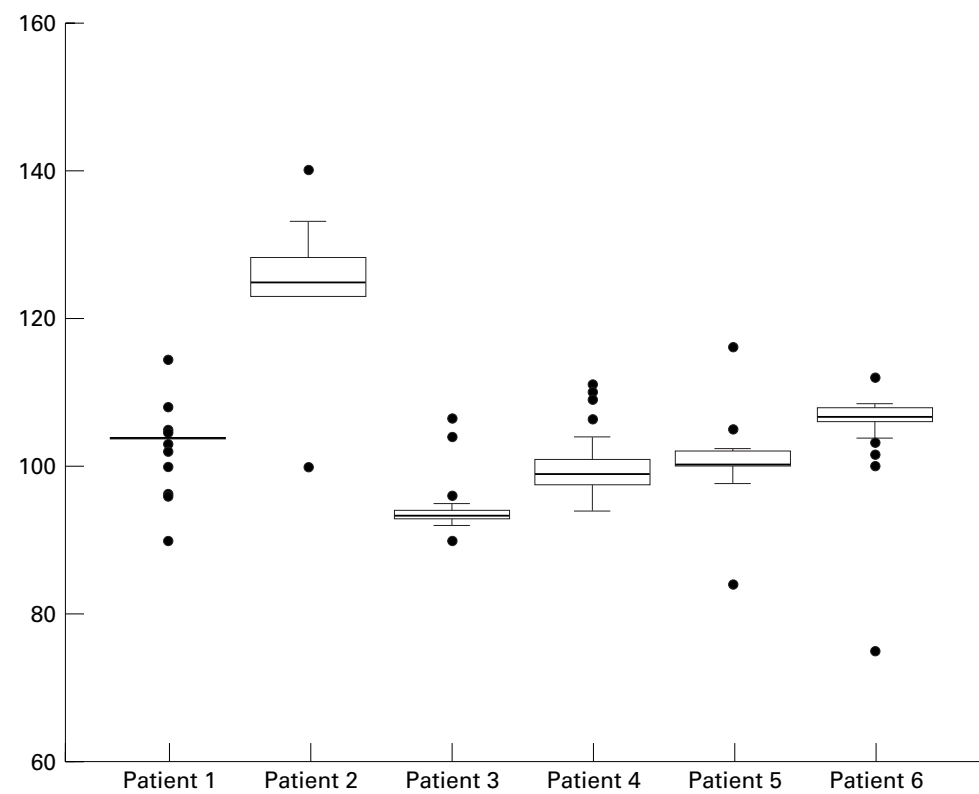

Figure 1 Results of interexaminer reliability of $\%$ WFH calculations. Medians are indicated by heavy black lines. Solid dots indicate outliers.

The expression of body weight as a percentage of ideal weight for height, gender, and age ( $\% \mathrm{WFH})$ has been suggested by the American Cystic Fibrosis Foundation as a useful measure of nutritional status in children with cystic fibrosis (CF). ${ }^{1}$ It is also used regularly as an outcome measure in $\mathrm{CF}$ research and as a measure of nutritional status in other areas of paediatrics including liver disease and oncology. ${ }^{2-5}$

\%WFH has the advantage of integrating both weight and height in the same measure and can easily be calculated. However, we have noticed variability between individuals in the calculation of \%WFH. A comprehensive literature review has failed to identify any work on the reliability of this measure. Therefore, the aim of this study was to investigate the reliability of using \%WFH as a measure of nutritional status.

\section{Methods}

Fifty two members of UK CF Dietitians Interest Group were sent a questionnaire examining the type of measures which they used to assess nutritional status. If they used \%WFH they were asked how frequently they did so and by which method.

Each dietitian was asked to complete $\% \mathrm{WFH}$ calculations based on the following details of six CF patients: date of birth, sex, height, weight, and the date on which the height and weight were taken. Growth charts were provided, as were the US CF Foundation instructions for calculating \%WFH:

1. Plot the height on the growth chart and determine height centile.

2. Determine the weight that corresponds to the same point on the centile chart as the height.

3. Divide actual weight by ideal weight, then multiply by 100 .

Eleven of 44 dietitians were contacted a month after receiving the first \%WFH calculation sheet, and asked to repeat the calculations based on the same data without referring back to their previous calculations.

Means, standard deviations, and ranges of the results obtained for each patient and for each dietitian were calculated.

\section{Results}

Forty four of the 52 dietitians completed the questionnaire. The majority $(35 / 44)$ used $\% \mathrm{WFH}$ as a measure of nutritional status. Of these, 14 assessed \%WFH annually and nine at each clinic visit. Height and weight centile measurements were recorded at each clinic by 41/44. The US CF Foundation method for calculating \%WFH was used by $22 / 35$ dietitians and the Cole Slide Rule was used by $9 / 35$.

$\% W F H$ calculations were completed by $42 / 52$. The range of estimates for each of the six patients varied by 16.5 to 40 percentage points (mean 27.8; fig 1). On average, $25 \%$ of the $\% \mathrm{WFH}$ calculations were more than two percentage points either side of the mean, and $8.5 \%$ were more than five percentage points either side of the mean.

Eleven of the dietitians completed the second set of calculations. The range of estimates for each of the six patients varied by between 13 and 24 percentage points (mean 16.8). For each of the 11 dietitians the average difference between the two sets of results was calculated and the mean of these was 3.4 percentage points (range 0.3 to 8.0 ). 


\section{Discussion}

The results indicate that \%WFH, when calculated by hand, is not a reliable measure of nutritional status because of the wide interand intraexaminer variation. Although this study was conducted with dietitians, many of whom use \%WFH regularly, other clinicians also use \%WFH to assess nutritional status. There are a number of areas where errors can be made, including the plotting of the patient's current actual weight, height, and ideal weight on the growth charts, and the calculation of $\% \mathrm{WFH}$ based on this information. In the USA and Canada a computer package designed specifically for the calculation of $\% \mathrm{WFH}$ is available (Pencharz P, personal communication). Such a package is not available in the UK and most dietitians in this study routinely calculate \%WFH by hand. Discussions with dietitians in Europe suggest that the situation is similar outside the UK.

In clinical practice the clinician needs to be aware of the unreliability of this measure when assessing a patient's current nutritional status and change in nutritional status with time. Although clinicians do not normally base their assessment of nutritional status on one measure and monitor a series of measures, including the child's progress on individual centile charts, they should be aware of the problems of using \%WFH when calculated by hand. One of the difficulties is in finding an alternative measure which integrates information about a child's height, weight, and age. Body mass index centile measures may be appropriate, but their reproducibility and reliability in children have not yet been established. The Cole Slide Rule was cited as the method of calculating $\% \mathrm{WFH}$ by $26 \%$ of dietitians. At the time of undertaking this research the Cole Slide Rule was based on the Tanner and Whitehouse growth charts which are out of date and are no longer appropriate for use in children in the UK. ${ }^{6}$ Since completing this research, the Cole Slide Rule has been updated and now calculates body mass index and percentage body mass index for age, using data from the current UK growth charts (Child Growth Foundation, personal comunication). We have been unable to identify any published work on the reliability of this slide rule.

In $\mathrm{CF}$ research \%WFH has been used as a nutritional outcome measure, recorded serially, or to identify a difference in nutritional status between two groups of patients. This study has highlighted the fact that whether $\% \mathrm{WFH}$ is repeated by the same dietitian, or calculated by a number of dietitians, it is an unreliable measure and should not be used in clinical research. The reliability of any measure proposed as an alternative to \%WFH should be assessed using a similar methodology to the one used here.

We thank the CF dietitians for their participation in the study and Professor Tim Cole for helpful comments on the manuscript.

1 Ramsey BW, Farrell PM, Pencharz P, and the Consensus Committee. Nutritional assessment and management in cystic fibrosis: a consensus report. Am $f$ Clin Nutr 1992;55:108-16.

2 Hanning RM, Blimkie CJR, Bar-Or O, Lands LC. Relationships among nutritional status and skeletal and respiratory muscle function in cystic fibrosis: does early dietary supplementation make a difference? Am f Clin Nutr 1993; 57:580-7.

3 Girardet JP, Tounian P, Sardet A, et al. Resting energy expenditure in infants with cystic fibrosis. F Pediatr Gastroenterol Nutr 1994;18:214-19.

4 Charlton CPJ, Buchanan E, Holden CE, et al. Intensive enteral feeding in advanced cirrhosis: reversal of malnutrition without precipitation of hepatic encephalopathy. Arch Dis Child 1992;67:603-7.

5 Uderzo C, Rovelli A, Bonomi M, et al. Nutritional status in untreated children with leukemia as compared with children without malignancy. I Pediatr Gastroenterol Nutr 1996;23:34-7.

6 Cole TJ, Donnet ML, Stanfield JP. Weight-for-height indices to assess nutritional status - a new index on a slide rule. Am f Clin Nutr 1981;34:1935-43. 